\title{
Antimicrobial Resistance Patterns of bacterial Septicaemia infecting infants in Mbita Subcounty, Western region of Kenya
}

\author{
Guyo H. Sora ${ }^{1,2,3}$, George Gachara ${ }^{2}$, Yoshio Ichinose ${ }^{3}$, Cyrus Kathieko ${ }^{1,3}$, Mohamed Karama ${ }^{4}$ \\ ${ }^{1}$ Centre for Microbiology Research, Kenya Medical Research Institute, Nairobi, Kenya \\ ${ }^{2}$ School of Medicine, Kenyatta University, Nairobi, Kenya \\ ${ }^{3}$ Nagasaki University Institute of Tropical Medicine, Kenya Research Station, Nairobi, Kenya; \\ ${ }^{4}$ Centre for Public Health Research, Kenya Medical Research Institute, Nairobi, Kenya. \\ Correspondence: Guyo H. Sora: Email: guyo.sora@yahoo.com
}

\begin{abstract}
Background: Gram positive bacteria such Escherichia coli, Group B Streptococcus coagulase-negative staphylococci, Staphylococcus aureus, and Gram-negative bacteria such as Klebsiella and Pseudomonas species are listed as some of the bacteria etiologies for pediatric septicemia. These bacteria are rapidly becoming multi drug resistant to penicillin (or aminopenicillin), gentamicin, the pragmatic antibiotic treatment regimens. Further, the ever-increasing burden of bacteria septicemia infection due to extended-spectrum $\beta$-lactamase (ESBL) producing Gram negative bacteria cumulatively presents a major health concern in the management and treatment of bacterial septicemia. In this study we present data on the prevalence and type of antimicrobial resistant patterns among children with bacterial septicemia in Mbita Sub county Hospital, Western region of Kenya.

Methods: Blood samples were obtained from 248 children whose parents/guardian consented. The bacterial isolation and characterization were done using the automated BACTEC 9240 system, conventional culture using morphology, Gram stain and biochemical identification. Further identification and resistant gene detection were determined using Polymerase Chain Reaction (PCR). Descriptive statistics were used to present data.

Results: Eighty-four (33.9\%) patients had septicemia where Staphylococcus epidermidis (28.6\%), S. aureus (13.1\%), Escherichia coli (13.1\%) and single Salmonella Paratyphi B, Citrobacter freundii, Gemella morbillorum, Klebsiella pneumoniae, Lactococcus lactis cremoris, Pantoea spp, and Pseudomonas putida were implicated. The majority of gram-negative bacteria were resistant to penicillin (Ampicillins) 100\%, 96.1\% to tetracyclin, 84.6\% to sulphonamides (Trimethoprim/sulfamethoxazole), 73.1\% Aminoglycosides (Gentamicin) 73.1\% and 19.2\% to Quinolone (Ciprofloxacin). For gram positive bacteria majority $96.7 \%$ were resistant to sulphonamides (Trimethoprim/sulfamethoxazole) followed by tetracycline $76.7 \%$, penicillin (Oxacilline) $73.3 \%$ and least resistant to Quinolone (Ciprofloxacin) 30\%. Various antimicrobial resistant genes mecA, SulII, blaTEM, TetA aac (3) were identified.

Conclusion. In this geographically defined region of Kenya, of the $33.9 \%$ children with septicemia, gram positive bacteria were the leading cause septicemia. High level resistance due to various resistant genes were seen all type of antibiotics by both Gram positive and negative bacteria. Rapid antibiotic resistant testing is encouraged for appropriate treatment and management of septicemia infection.
\end{abstract}

Keywords: bacterial Septicemia, Epidemiology, Children under five, South Nyanza, Kenya

DOI: $10.7176 / J N S R / 10-10-07$

Publication date:May $31^{\text {st }} 2020$

\section{Introduction}

Globally, bloodstream infections and sepsis are major causes of morbidity and mortality. Epidemiological data from high-income countries reported that 31.5 million cases of sepsis and 5.3 million sepsis attributable deaths occur annually (Fleischmann et al., 2015). Data from low-and-middle-income countries (Reinhart et al., 2017). Previous estimates, shows a range of 380 000-2 000000 annual cases of neonatal sepsis in sub-Saharan Africa and 270000 annual associated deaths, highlight the substantial burden of disease (Seale et al., 2010; Sepsis Alliance, 2016). Most studies on sepsis and bloodstream infections report an increasing incidence over the last two decades, particularly among the immunocompromised, multimorbid, and elderly patients, or due to failure of empiric antibiotic regimens as result of antimicrobial resistance (Goto \& Al-Hasan, 2013; Cassini et al. 2019). 
Children in developing countries are the most susceptible segment of the population, laying emphasis on the need for extraordinary consideration given that bloodstream infections and sepsis is the major cause of child mortality and morbidity (Kissoon et al., 2011). Invasive bacteria contribute largely to the etiologies of septicemia in African children (Jacob et al.,2009). Some of the bacteria etiologies for pediatric septicemia reported in varying prevalence in sub-Saharan Africa include Escherichia coli, Group B Streptococcus coagulase-negative staphylococci, Staphylococcus aureus, and Gram-negative organisms such as Klebsiella and Pseudomonas species (Zaidi et al., 2009). The rapid spread of multi drug resistant bacterial pathogens is likely to complicate treatment of bacteria septicemia (Taxt et al., 2020). The widely embraced pragmatic antibiotic treatment regimens for sepsis based on penicillin (or aminopenicillin) in combination with gentamicin are faced with emergence of multidrug resistance jeopardizing their utility (Fuchs et al., 2018).

Of importance, the ever-increasing burden of bacteria septicemia infection due to extended-spectrum $\beta$-lactamase (ESBL) producing Gram negative bacteria represents a major health concern (Taxt et al., 2020). These bacteria, mainly Escherichia coli and Klebsiella pneumoniae, are not only resistant to all penicillins and third generation cephalosporins, but also frequently express co-resistance to gentamicin (Taxt et al., 2020). Inevitably, treatment failure accompanied by increased treatment cost and fatality are likely to occur especially in developing nations. The increasing prescription of third and fourth generation - last-resort antibiotics such as carbapenems as initial antibiotic treatment of sepsis is likely to worsen sepsis management in developing nations most of which sell these antibiotics without the need for doctors' prescription (Taxt et al., 2020). Increased emergence and spread of AMR are likely to be a big challenge in the near future especially in resource limited nations. Inevitably, rapid diagnosis of bacteria septicemia is crucial in the prescription of appropriate antimicrobial therapy likely to lower morbidity and mortality (Buehler et al., 2016). In this study we present data on the prevalence and type of antimicrobial resistant patterns among children with bacterial septicemia in Mbita Sub county Hospital, Western region of Kenya.

\section{METHODOLOGY}

\section{Study setting and design}

This was a descriptive hospital based cross-sectional study conducted between 2019 and 2020 among children presenting with symptoms suggestive of septicemia as described by the WHO (Bataar et al., 2010), attending Mbita District Hospital in Nyanza Kenya. Using the formula for estimating the population proportion with specified relative precision described by Lemeshow et al (1990) setting the $\alpha$ at 0.05 , and prevalence of bacterial septicemia of $76 \%$ (WHO, 2014), a total of 281 patients were recruited to achieve 0.95 power.

\section{Recruitment and ethical approvals}

This study recruited patients if: 1 . Had clinical symptoms suggestive of septicemia as defined by the WHO. 2 . Attending/admitted at Mbita District Hospital. 2. Age between 1 day to 120 months. 3. Parents/guardian providing informed consent. Study patients were then consecutively enrolled till the desired number was achieved. This study was approved by Ethical Review Committee of Kenyatta University before commencing to field activities. Each participant signed a form of informed consent

\section{Laboratory analysis}

Blood sample collection and transportation: From each of the participants enrolled, about 2-5ml (children) blood samples were collected aseptically in aerobic and anaerobic blood culture bottles BD (Becton Dickinson, US). The sampling bottles were appropriately labelled in line with pathological/request forms details i.e. name, sample code, date, time and location of the hospital and patients. The blood samples collected aseptically into appropriate blood culture tubes were packed in primary cases and secondary cases according to the WHO guideline for transporting infectious materials. The samples were maintained in upright position in a cooler box and transported to NUITM-KEMRI Biosafety level 2 laboratory at Mbita for processing.

\section{Microbiological analysis}

Samples were incubated at $37^{\circ} \mathrm{C}$ for at least 3 weeks in the BACTEC 9270 (Becton Dickinson, US) automated machine. Generally, the BACTEC indicates signals for any positive culture wells. All positive culture bottle was taken to the Biosafety level III laboratory where they were sub cultured using sterile and disposable loops on basic, differential, selective media (Oxoid type) and other appropriate media like blood agar, chocolate blood agar (CBA), Xylose lysine deoxycholate (XLD), DHL, Salmonella-Shigella agar (SS), and Bromol thymol blue (BTB) 
for other etiological agents. All plates were incubated at $37^{\circ} \mathrm{C}$ for $18-24 \mathrm{hrs}$. Blood agar, Brucella agar and CBA were incubated in the presence of $5-10 \% \mathrm{CO} 2$. The suspected colonies were examined and characterized by their morphology, Gram stain, biochemical identification (Sahin, et al., 2008). All the identified and clinically significant isolates were purified, accurately labelled and stored in $15-40 \%$ glycerol, at $-80^{\circ} \mathrm{c}$ for any future work or references.

\section{Antibiotic susceptibility testing}

Isolates were tested for their antimicrobial susceptibility by the Kirby-Bauer disk diffusion method according to the Clinical and Laboratory Standards Institute (CLSI) guidelines (CLSI, 2018). The drugs tested included; cefoxitin (CTX), streptomycin (STR), tetracycline (TET), Ampicillin (AMP), chloramphenicol (CHLO), ciprofloxacin (CIP), Nalidixic acid (NA), gentamicin (GEN), trimethoprim-sulfamethoxazole (SXT). The antimicrobial susceptibility was classified using the CLSI guidelines, as susceptible, intermediate, or resistant to each antibiotic. In addition, isolates were also classified as either non-susceptible (including both intermediate and resistant isolates) or susceptible. Isolates found to be non-susceptible or resistant to three or more antimicrobial categories were classified as multidrug resistant (MDR).

\section{Detection of resistant genes}

Plasmid DNA extraction: Profiling for R-plasmids and screening for the presence of previously reported resistance gene TetA gene, StrA gene Str B gene, Sul2gene, Amp C gene, Gyr A gene, CatAl gene were done with modification as described by Szczepanowski et al., (2009). The representative of all bacterial isolates belonging to various antibiograms were selected and sub-cultured on Mueller-Hinton media overnight at $37^{\circ} \mathrm{C}$ and the plasmid DNA extracted using the alkaline lysis method described by Sambrook et al., (2001). Briefly, the plasmid DNA was extracted from 2-ml overnight cultures grown in Luria-Bertani (LB) broth (Difco Laboratories, Becton Dickinson, Sparks, MD). Plasmid sizes were determined using electrophoresis in comparison with plasmids from the reference strains E. coli V517 and E. coli 39R861 (Macrina, 1979).

PCR and amplicon detection. The reaction mix of the PCR was composed of about $100 \mathrm{ng}$ total plasmid DNA as template, $2.5 \mu \mathrm{l} \mathrm{PuReTaq} \mathrm{Ready-To-Go} \mathrm{PCR} \mathrm{beads} \mathrm{and} 0.5 \mu \mathrm{M}$ of each primer (Table 1) and filled up to $25 \mu \mathrm{l}$ with sterile double-distilled water. The PCR was performed using Bio-Rad iCycler Thermal Cycler (Champaign, US). The initial step of the reaction was denaturation of DNA at $94{ }^{\circ} \mathrm{C}$ for $4 \mathrm{~min}$. This step was followed by 35 cycles composed of $1 \mathrm{~min}$ denaturation at $94{ }^{\circ} \mathrm{C}, 1 \mathrm{~min}$ annealing at $58{ }^{\circ} \mathrm{C}$ and $45 \mathrm{~s}$ polymerization at $72{ }^{\circ} \mathrm{C}$. The final polymerization step was performed for $10 \mathrm{~min}$ at $72{ }^{\circ} \mathrm{C}$. The amplicons were analyzed by gel electrophoresis (in $1 \%$ agarose in Tris/ $\mathrm{HCl} /$ acetate buffer), stained with ethidium bromide and visualized under UV light.

\section{Data analysis}

Descriptive statistics frequencies (\%), standard deviation, mean, and medians (interquartile ranges at $25 \%$ and $27 \%$ ) were used to present data. Where applicable, test for significance was done using Fisher's exact test or Chisquare at the significance level of P 0.05. All statistical analyses were performed using STATA v 13 (StataCorp LP, College Station, TX, USA). 
Table 1 gene identities, primer sequences, molecular sizes and the source

\begin{tabular}{|c|c|c|c|c|}
\hline Target & Forward & Reverse & $\begin{array}{l}\text { Size- } \\
\text { bp }\end{array}$ & Reference \\
\hline StrA & GCTGGATAGGTTAAGGGCGG & CTCTATGGGCACTGTCCACATTG & 383 & $\begin{array}{l}\text { Hochhut et al., } \\
2001\end{array}$ \\
\hline Sul2 & AGGGGGCAGATGTGATCGAC & TGTGCGGATGAAGTCAGCTCC & 625 & $\begin{array}{l}\text { Hochhut et al., } \\
2001\end{array}$ \\
\hline TetA & ATAAAATTCTTGAAGACGAAA & GACAGTTACCAATGCTTAATC & 1080 & Weill et al., 2004 \\
\hline $\begin{array}{l}C T X- \\
M\end{array}$ & AAA AAT GAT TGA AAG GTG GT & CAG CGC TTT TGC CGT CTA AG & & $\begin{array}{l}\text { Karunakaran et al., } \\
2012\end{array}$ \\
\hline CAtA1 & AAGCGAACGA & GGAAGTAAAA & & $\begin{array}{l}\text { Hochhut et al., } \\
2001\end{array}$ \\
\hline$A m p C$ & AACACACTGATTGCGTCTGAC & CTGGGCCTCATCGTCAGTTA & 1870 & $\begin{array}{l}\text { peres-peres et al., } \\
2002\end{array}$ \\
\hline Gyr $A$ & TTAATGATTGCCGCCGTCGG & TACACCGGTCAACATTGAGG & 648 & $\begin{array}{l}\text { Jaktaji \& Mohiti, } \\
2010\end{array}$ \\
\hline mecA & $\begin{array}{l}\text { GTA GAA ATG ACT GAA CGT } \\
\text { CCGATAA }\end{array}$ & $\begin{array}{l}\text { CCA ATT CCA CAT TGT TTC } \\
\text { GGTCTA A }\end{array}$ & 533 & $\begin{array}{l}\text { Wielders et al., } \\
2002\end{array}$ \\
\hline
\end{tabular}

StrA - Streptomycin resistance; Sul2 - sulfamethoxazole resistance; TetA - tetracycline resistance; CTX-M Ceftriaxone; CAtAl gene - Chloramphenicol; AmpC-Augumentin; Gyr A - Ciprofloxacin; mecA Gene Oxacillin/methicillin

\section{RESULTS}

Characteristics of study patients

All the data was available for 248 out of recruited 281 children. The mean ( \pm standard deviation - SD) age of the participants was $27.93( \pm 20.6)$ months with $30.6 \%$ of them aged between 1 to 12 months. The majority of the patients $50.8 \%$ were males, $48 \%$ from Rusinga locality and $91.9 \%$ HIV negative. The mean body temperature for the patients $38^{\circ} \mathrm{C}( \pm 20.5)$ ranging between 37 to $40^{\circ} \mathrm{C}$. There were $58.9 \%$ patients with body temperatures above $37.6{ }^{\circ} \mathrm{C}$. The mean $\mathrm{WBC}$ of the patients was $17720.9 \mathrm{Cells} / \mathrm{ml}( \pm 8929.1) \mathrm{Cells} / \mathrm{ml}$ ranging between 12075 to 22450 Cells $/ \mathrm{ml}$. about $25.4 \%$ of them had WBC above the normal levels of $10501 \mathrm{cells} / \mathrm{ml}$. The mean respiratory rate $(\mathrm{RR})$ of the patients was $30.6( \pm 10.6)$ breaths/min ranging between 18 to 96 breaths $/ \mathrm{min}$ with $71.4 \%$ having RR between 20 and 30 breaths/min. Co-infection/complications among the study patients included: malaria reported in $83(33.5 \%)$ of the patients followed by respiratory illnesses $33(13.3 \%)$, Hematologic diseases $31(12.5 \%)$, Gastrointestinal disorders $27(10.9 \%)$, malnutrition and meningitis in $9(3.6 \%)$ each. There were 6 (2.4\%) patients who had Nervous system diseases, 5(2.1\%) with Ear nose and throat infections and $2(0.8 \%)$ with HIV. There were $20(8.1 \%)$ patients who reported no other co-infection.

\section{Etiology of septicemia among study patients}

A total of 18 different etiological agents were identified in this study from 84 of the 248 (33.9\%) children who had septicemia. The most common causative agent of septicemia was Staphylococcus epidermidis (28.6\%) followed by $S$. aureus and $E$. coli each at $13.1 \%$. Others included $P$. aeroginosa (10.7\%), S. typhimurium (8.3\%), S. hemolyticus (4.8\%) among others (Figure 1). 


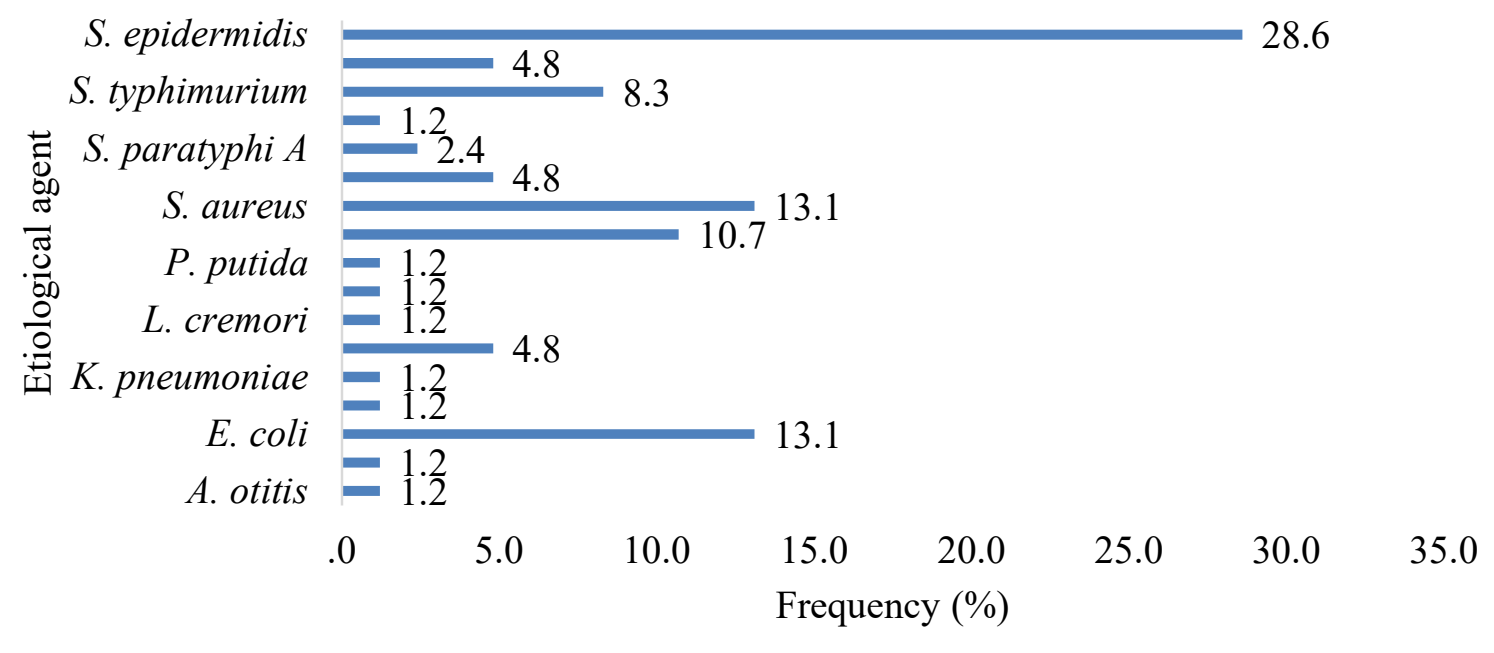

Figure 1: Frequency of etiological agents of septicemia among the study patients

\section{Antimicrobial resistant patterns of bacterial septicemia etiological agents}

Table 1 summarizes the drug susceptibility patterns for bacteria causing septicemia among study participants. Susceptibility testing showed that majority of gram-negative isolates were resistant to penicillin (Ampicillins) $100 \%$ followed by tetracycline $96.1 \%$, sulphonamides (Trimethoprim/sulfamethoxazole) $84.6 \%$, Aminoglycosides (Gentamicin) 73.1\% while they were least resistant to Quinolone (Ciprofloxacin) 19.2\%. For gram positive bacteria majority $96.7 \%$ were resistant to sulphonamides (Trimethoprim/sulfamethoxazole) followed by tetracycline 76.7\%, penicillin (Oxacilline) $73.3 \%$ and least resistant to Quinolone (Ciprofloxacin) $30 \%$.

Table 1: Distribution of drug susceptibility pattern of bacteria causing septicemia

\begin{tabular}{|c|c|c|c|c|c|c|c|}
\hline \multicolumn{8}{|c|}{ Drug susceptibility patterns for gram negative isolates } \\
\hline \multirow[t]{3}{*}{ Antibiotic class } & \multirow[t]{3}{*}{ Antibiotic tested } & \multicolumn{4}{|c|}{ Susceptibility pattern } & & \\
\hline & & \multicolumn{2}{|c|}{ Sensitive } & \multicolumn{2}{|c|}{ Intermediate } & \multicolumn{2}{|c|}{ Resistant } \\
\hline & & $\mathbf{N}$ & $\%$ & $\mathbf{N}$ & $\%$ & $\mathbf{N}$ & $\%$ \\
\hline \multirow[t]{2}{*}{ Penicillins } & Ampicillin & 0 & 0 & 0 & 0 & 26 & 100 \\
\hline & Amoxicillin/Clavulanate & 16 & 61.5 & 1 & 3.9 & 9 & 34.6 \\
\hline \multirow[t]{2}{*}{ Cephalosporin } & Cefuroxime & 7 & 26.9 & 1 & 3.9 & 18 & 69.2 \\
\hline & Ceftriaxone & 10 & 38.5 & 2 & 7.5 & 14 & 53.9 \\
\hline Tetracyclines & Tetracycline & 0 & 0 & 1 & 3.9 & 25 & 96.1 \\
\hline Quinolone & Ciprofloxacin & 20 & 76.9 & 1 & 3.9 & 5 & 19.2 \\
\hline Aminoglycosides & Gentamicin & 6 & 23.1 & 1 & 3.9 & 19 & 73.1 \\
\hline Sulfonamides & Trimethoprim/sulfamethoxazole & 3 & 11.5 & 1 & 3.9 & 22 & 84.6 \\
\hline \multicolumn{8}{|c|}{ Drug susceptibility patterns for gram positive isolates } \\
\hline \multirow[t]{2}{*}{ Penicillins } & Oxacillin & 8 & 26.7 & 0 & 0 & 22 & 73.3 \\
\hline & Amoxicillin/Clavulanate & 14 & 46.7 & 0 & 0 & 16 & 53.3 \\
\hline Macrolides & Erythromycin & 16 & 53.3 & 0 & 0 & 14 & 46.7 \\
\hline Tetracyclines & Tetracycline & 6 & 20 & 1 & 3.3 & 23 & 76.7 \\
\hline Quinolone & Ciprofloxacin & 20 & 66.7 & 1 & 3.3 & 9 & 30 \\
\hline Aminoglycosides & Gentamicin & 14 & 46.7 & 4 & 13.3 & 12 & 40 \\
\hline Sulfonamides & Trimethoprim/sulfamethoxazole & 0 & 0 & 1 & 3.3 & 29 & 96.7 \\
\hline
\end{tabular}




\section{Drug resistant genes among the multi drugs resistant bacterial etiological agents}

The number of multi-drug resistant bacterial isolates included 4 Staphylococcus epidermidis, 4 S. aureus, 7 E. coli (4 Enterotoxigenic and 3 Enteropathogenic E. coli), 4 S. paratyphi (3 S. paratyphi A and 1 S. paratyphi B), $3 S$. typhimurium and $3 P$. aeruginosa

S. aureus and S. epidermidis: Plasmids isolated from both $S$. epidermidis and $S$. aureus species harbored resistance genes, mecA and SulII, highlighting their role in dissemination of antibiotic resistance. High frequency of resistance was observed for Sulfamethoxazole-Trimethoprim (87.5\%), Oxacilin (75.0\%), Erythromycin (62.5\%) and Amoxyclavulanic acid (50.0\%). All the isolates were multiply resistant to between two to six antibiotics. One of the $S$. epidermidis did not carry any plasmid but showed phenotypic resistance to Oxacilin, Amoxyclavulanic acid, Sulfamethoxazole-Trimethoprim and Tetracycline (Table 2).

E. coli: Plasmids isolated from the E. coli isolates studied harbored resistance genes, blaTEM, SulII, and TetA. High frequency of resistance was observed for Sulfamethoxazole-Trimethoprim (100\%), Ampicillin (100\%), Tetracycline (100\%) and Gentamycin $(85.7 \%)$. All the isolates were multiply resistant to between four and eight antibiotics. However, there was no apparent relationship between carriage of plasmids and antimicrobial resistance (Table 2).

Table 2. Distribution of resistant genes among MDR $S$. epidermidis, $S$. aureus and pathogenic $E$. coli

\begin{tabular}{|c|c|c|c|c|c|c|c|c|}
\hline \multirow[t]{2}{*}{ Sample } & \multirow[t]{2}{*}{ Isolate } & \multirow[t]{2}{*}{$\begin{array}{c}\text { No. of } \\
\text { plasmids }\end{array}$} & \multicolumn{4}{|c|}{$\begin{array}{c}\text { Approximate sizes of the } \\
\text { plasmids }(\mathrm{Kb})\end{array}$} & \multirow[t]{2}{*}{ Pattern of drug resistance } & \multirow[t]{2}{*}{$\begin{array}{c}\text { Antimicrobial genes } \\
\text { detected }\end{array}$} \\
\hline & & & 1500 & 3000 & 4000 & $>10000$ & & \\
\hline $\mathrm{MDH} / \mathrm{BLD} / 54$ & S. aureus & 2 & 0 & 0 & 0 & 2 & OX, Amoclav, Sxt, Tet, Ery & mecA \\
\hline $\mathrm{MDH} / \mathrm{BLD} / 55$ & S. aureus & 1 & 0 & 0 & 0 & 1 & OX, Gen, Tet, Ery & mecA \\
\hline $\mathrm{MDH} / \mathrm{BLD} / 15$ & S. aureus & 1 & 0 & 0 & 0 & 1 & Cip, Tet, Sxt, Ery & SulII \\
\hline $\mathrm{MDH} / \mathrm{BLD} / 135$ & S. aureus & 1 & 0 & 0 & 0 & 1 & OX, Sxt & mecA \\
\hline $\mathrm{MDH} / \mathrm{BLD} / 157$ & S. epidermidis & 0 & 0 & 0 & 0 & 0 & OX, Amoclav, Sxt, Tet & none \\
\hline MDH/BLD/62 & S. epidermidis & 2 & 0 & 0 & 0 & 2 & Amoclav, Sxt, Ery & $m e c A$ \\
\hline $\mathrm{MDH} / \mathrm{BLD} / 216$ & S. epidermidis & 2 & 0 & 0 & 0 & 2 & OX, Cip, Gen, Tet, Sxt & $m e c A$ \\
\hline MDH/BLD/104 & S. epidermidis & 2 & 0 & 0 & 0 & 2 & OX, Amoclav, Cip, Sxt, Tet, Ery & $m e c A$ \\
\hline $\mathrm{MDH} / \mathrm{BLD} / 183$ & E. coli (EPEC) & 3 & 1 & 1 & 0 & 1 & Amp, Gen, Sxt, Tet & blaTEM, SulII \\
\hline $\mathrm{MDH} / \mathrm{BLD} / 222$ & E. coli (EPEC) & 5 & 1 & 1 & 0 & 3 & Amp, Amoclav, Gen, Sxt, Tet & SulII, TetA \\
\hline $\mathrm{MDH} / \mathrm{BLD} / 229$ & E. coli (EPEC) & 5 & 1 & 1 & 0 & 3 & Amp, Gen, Sxt, Tet & blaTEM, SulII, TetA \\
\hline MDH/BLD/142 & E. coli (ETEC) & 4 & 1 & 1 & 0 & 2 & Amp, Amoclav, Sxt, Tet & blaTEM, SUlII, TetA \\
\hline $\mathrm{MDH} / \mathrm{BLD} / 163$ & E. coli (ETEC) & 3 & 1 & 1 & 0 & 1 & Amp, Gen, Sxt, Tet & blaTEM, SulII, TetA \\
\hline $\mathrm{MDH} / \mathrm{BLD} / 215$ & E. coli (ETEC) & 3 & 1 & 1 & 0 & 1 & Amp, Gen, Sxt, Tet & blaTEM, SUlII \\
\hline $\mathrm{MDH} / \mathrm{BLD} / 156$ & E. coli (ETEC) & 4 & 1 & 1 & 0 & 2 & Amp, Amoclav, Cfm, Cfx, Gen, Cip, Sxt, Tet & blaTEM, SulII, TetA \\
\hline
\end{tabular}

Where Ox-Oxacillin; Amoclav-Amoxicillin/Clavulanate; Sxt-Trimethoprim/sulfamethoxazole; Tet-

Tetracycline; Ery-Erythromycin; Gen-Gentamicin; Cip-Ciprofloxacin; mec A-Methicillin-resistant

Staphylococcus aureus; blaTEM - Nonspecific TEM B-Lactamase; TetA-Tetracycline Resistant gene A; SulII -

Sulfonamide resistance gene

Pseudomonas spp : The antimicrobial gene, blaTEM, was detected in five of the plasmids while four of the plasmids carried SulII, TetA and aac (3) antimicrobial resistance genes. High frequency of resistance was observed for Tetracycline (100\%), Ampicillin (100\%), Cefixime (80\%), Cetriaxone (80\%), Amoxyclavulanic acid (80.0\%), Gentamicin (60.0\%) and Sulfamethoxazole-Trimethoprim $(60 \%)$, All the isolates were multiply resistant to between to two to six antibiotics.

Salmonella spp: Plasmids isolated from the Salmonella isolates studied harbored resistance genes, blaTEM, SulII, aac (3) and TetA highlighting their role in transmission of resistance. High frequency of resistance was observed for Sulfamethoxazole-Trimethoprim (100\%), Ampicillin (100\%), Tetracycline (100\%), Cefixime (100\%), Gentamycin (100\%) and Ceftriaxone (57.1\%). All the isolates were multiply resistant to between to four to seven antibiotics. One of the Salmonella did not carry any plasmid but showed phenotypic resistance to Cefixime, Ampicillin, Sulfamethoxazole-Trimethoprim and Tetracycline. However, there was no apparent relationship between carriage of plasmids and antimicrobial resistance. 
Table 3. Distribution of resistant genes among MDR $S$. paratyphi and $S$. typhimurium

\begin{tabular}{|c|c|c|c|c|c|c|c|c|}
\hline \multirow[t]{2}{*}{ Sample } & \multirow[t]{2}{*}{ Isolate } & \multirow[t]{2}{*}{$\begin{array}{c}\text { No. of } \\
\text { plasmids }\end{array}$} & \multicolumn{4}{|c|}{$\begin{array}{c}\text { Approximate sizes of the } \\
\text { plasmids }\end{array}$} & \multirow[t]{2}{*}{ Pattern of drug resistance } & \multirow[t]{2}{*}{ Antimicrobial genes detected } \\
\hline & & & 1500 & 3000 & 4000 & $>10000$ & & \\
\hline MDH/BLD/093 & S. paratyphi $\mathrm{B}$ & 4 & 0 & 1 & 1 & 2 & Amp, Cfm, Gen, Sxt, Tet & blaTEM, SulII, TetA, aac(3) \\
\hline MDH/BLD/219 & S. paratyphi A & 3 & 0 & 1 & 1 & 1 & Amp, Cfm, Cfx, Gen, Sxt, Tet & blaTEM, SulII, TetA, aac(3) \\
\hline $\mathrm{MDH} / \mathrm{BLD} / 238$ & S. paratyphi A & 5 & 0 & 1 & 1 & 3 & Amp, Cfm, Cfx, Gen, Sxt, Tet & blaTEM, SulII, TetA, aac(3) \\
\hline $\mathrm{MDH} / \mathrm{BLD} / 203$ & S. paratyphi A & 3 & 0 & 1 & 1 & 1 & Amp, Cfm, Cfx, Gen, Sxt, Tet & blaTEM, SulII, TetA, aac(3) \\
\hline MDH/BLD/133 & S. Typhimurium & 0 & 0 & 0 & 0 & 0 & Amp, Cfm, Gen, Sxt, Tet & - \\
\hline MDH/BLD/134 & S. Typhimurium & 5 & 0 & 1 & 1 & 3 & Amp, Cfm, Gen, Sxt, Tet & blaTEM, SulII, TetA, aac(3) \\
\hline MDH/BLD/189 & S. Typhimurium & 3 & 0 & 1 & 1 & 1 & Amp, Cfm, Cfx, Gen, Sxt, Tet & SulII, TetA, aac(3) \\
\hline $\mathrm{MDH} / \mathrm{BLD} / 11$ & P. aeruginosa & 1 & 0 & 0 & 0 & 1 & Amp, Amoclav, Cfm, Cfx, Cip, Sxt, Tet & blaTEM, SulII, TetA, aac(3) \\
\hline $\mathrm{MDH} / \mathrm{BLD} / 4$ & P. aeruginosa & 2 & 0 & 0 & 0 & 2 & Amp, Amoclav, Cfx, Gen, Sxt, Tet & blaTEM, SulII, TetA, aac(3) \\
\hline $\mathrm{MDH} / \mathrm{BLD} / 8$ & P. aeruginosa & 2 & 0 & 0 & 0 & 2 & Amp, Amoclav, Cfm, Cfx, Cip, Tet & blaTEM, SulII, TetA \\
\hline $\mathrm{MDH} / \mathrm{BLD} / 35$ & P. aeruginosa & 1 & 0 & 0 & 0 & 1 & Amp, Cfm, Cfx, Gen, Sxt, Tet & blaTEM, aac(3) \\
\hline $\mathrm{MDH} / \mathrm{BLD} / 175$ & $P$ aeruginosa & 1 & 0 & 0 & 0 & 1 & Amp, Amoclav, Cfm, Gen, Cip, Tet & blaTEM, SulII, TetA, aac(3) \\
\hline
\end{tabular}

Where: Amp-Ampicillin; Cfm-Cefuroxime; Cfx-Ceftriaxone; Amoclav-Amoxicillin/Clavulanate; SxtTrimethoprim/sulfamethoxazole; Tet-Tetracycline; Gen- Gentamicin; Cip-Ciprofloxacin; blaTEM - Nonspecific TEM $\beta$-Lactamase; TetA-Tetracycline Resistant gene A; SulII - Sulfonamide resistance gene; aac(3) Aminoglycoside acetyltransferase gene

\section{Discussions}

Early diagnosis and early appropriate treatment are crucial in cases of bacterial blood infection. In severe sepsis, the case fatality increases for each hour the antibiotic treatment is delayed (Ferrer et al., 2014). Therefore, empirical antibiotic treatment has to be initiated before the results of blood cultures are available. However, as infections with resistant microbes is an escalating problem worldwide, it is increasingly challenging to maintain appropriate antibiotic regimens for initial empiric therapy (Nathan and Cars, 2014; WHO, 2014). Resistant pathogenic bacteria are found frequently worldwide (WHO, 2014). Studies have shown that most developing countries are home to a number of risk factors for the emergence and spread of antibiotic resistance. Misuse of antibiotics, over-the-counter and parallel market access, and counterfeit or poor-quality drugs, combined with substandard hygiene and living conditions, are the driving forces behind the emergence and spread of antibiotic resistance (Kelesidis et al., 2007). The potential for the development and rapid spread of new forms of resistance is highlighted by the recent worldwide proliferation of NDM-1-producing Enterobacteriaceae. The gene, which confers resistance to carbapenems, originated in India in 2009, and since 2010 NDM-1-producing Enterobacteriaceae have been reported in North America, Europe, and Asia (Kumarasamy et al., 2010). The WHO has recently heightened awareness of this pressing issue with calls for action to contain antibiotic resistance on a global scale (WHO, 2014). In Kenya, a regimen containing penicillin and gentamicin, plus metronidazole if an anaerobic infection is suspected, has been recommended for more than thirty years in sepsis with unknown focus and etiology (Lee et al., 2014). In recent years, however, increasing numbers of infections with methicillin-resistant S. aureus (MRSA), extended-spectrum beta-lactamase producing Enterobacteriaceae, and vancomycin resistant enterococci have been detected (Lee et al., 2014). Selection of inherently resistant microbes due to antibiotic use is also a challenge. Updated knowledge about the distribution of microbes in serious infection and their resistance against antimicrobial agents is needed to ensure appropriate empiric antimicrobial treatment regimens. It is also important to identify subgroups in which tailored regimens are required. This was the basis important aspect of this study

\section{S. aureus}

The three out of four $S$. aureus isolates causing septicemia had the mecA antimicrobial gene while one carried SulII antimicrobial gene. One of the four isolates were multi-drug resistant to five different drugs (Oxacillin, Amoxicillin/Clavulanate, Trimethoprim/sulfamethoxazole, Tetracycline and Erythromycin). Two isolates were resistant to four different drugs and one isolate to two different drugs. Our study further shows that $75 \%$ of the $S$. aureus were Methicillin resistance. The $S$. aureus with varying antibiotic profiles have been associated with sepsis in other settings. In Kilifi District Hospital on the Kenyan coast Talbert et al., (2010) reported that all the Staphylococcus aureus blood culture isolates were susceptible to methicillin. The S. aureus causing neonatal sepsis in Tikur Anbessa University Hospital, Ethiopia; showed high-level resistance to ampicillin, cefiriaxone, cephalothin, chloramphenicol, and gentamicin (Shitaye et al., 2010). In Pakistan, Mir et al., (2011) all the 4.2\% reported $S$. aureus were methicillin-resistant S. aureus (MRSA). Dramowski et al., (2015) the $S$. aureus etiology of septicemia $65 \%$ of them were Methicillin-resistant. About $40 \%$ of all lethal cases of sepsis caused by Staphylococcus spp among children admitted at the Hospital, affiliate of Vilnius University Hospital in in 
Lithuania was resistant to Methicillin (Bobelytė, 2017). Our study and other showing significantly high prevalence of multidrug resistant $S$. aureus is worrying given bacteraemias due $S$. aureus are difficult to treat and is associated with 29-63\% mortality (Kaasch et al., 2014; Fortuin-de Smidt et al., 2015). The emergence of new CA-MRSA strains in the community has huge implications on patient treatment (David et al., 2010).

\section{S. epidermidis}

There were 3 out of 4 (75\%) S. epidermidis that had antimicrobial gene, mecA and only one isolate did not carry any plasmid. All four $S$. epidermidis were resistant to 3 to 6 different antibiotics. The strain that had no plasmid containing antimicrobial gene, mecA showed phenotypic resistance to Oxacilin, Amoxyclavulanic acid, Sulfamethoxazole-Trimethoprim and Tetracycline. Studies continue to report the importance of Coagulasenegative staphylococci as among the leading cause of nosocomial sepsis, especially in neonates (Marchant et al., 2013; Becker et al., 2014). Coagulase-negative staphylococci sepsis most often originates from the infection of indwelling medical devices, such as in catheter-related bloodstream infections or central line-associated blood stream infections (Vassallo et al., 2015). Most prominent among Coagulase-negative staphylococci infections are those due to the skin commensal S. epidermidis (Vassallo et al., 2015). However, the bacterial factors contributing to the development of sepsis, in particular in CNS, are poorly understood. Most S. epidermidis blood infections are caused by methicillin-resistant strains, with methicillin resistance rates even exceeding those found among $S$. aureus (Qin et al., 2017).

Methicillin-resistant $S$. epidermidis isolates from patients are cross-resistant to all b-lactam antibiotics, even though some might be susceptible to certain b-lactam agents by in vitro testing (Raad et al., 1998). Therefore, this pattern of resistance is similar to what has already been established with methicillin-resistant $S$. aureus. The prevalence of resistance has increased rapidly over the last three decades and has been attributed to the selection effect of the increasing use of b-lactam antibiotics. Studies have demonstrated an increase in the prevalence of resistant $S$. epidermidis in hospitals when isolates from 1964 were compared with isolates from 1986 or resistance and plasmid profiles (Raad et al., 1998). This resistance was plasmid-mediated. Some investigators attributed resistance in S. epidermidis to the action of the mecA gene (Raad et al., 1998). However, Mempel et al., (1994) demonstrated that $S$. epidermidis isolates could be methicillin resistant and lack mec $A$ transcription.

\section{E. coli}

The seven pathogenic E. coli species harbored three types of antimicrobial gene, SulII, blaTEM and TetA. One pathogenic ETEC was resistant to 8 different antibiotics, 3 ETEC were multi-drug resistant to 4 different antibiotics. One EPEC was resistant to 5 different antibiotics while the remaining 2 EPEC were resistant to four different antibiotics. Similar studies exist showing high level antibiotic resistance to $E$. coli species isolated from neonatal septicemia cases. Studies showed resistance to penicillin/ampicillin ranging from 55\% among E. coli isolates in Georgia (Macharashvili et al., 2009) to 100\% among E. coli isolates in Uganda (Mugalu et al., 2006). Resistance to gentamicin among E. coli ranged from 0\% in Pakistan (Mir et al., 2011), 21.7\% in South Africa (Dramowski et al., 2015), 67 in India (Jyothi et al., 2013). In Lithuania, sepsis-causing pathogens E. coli was characterized by the development of increasing antibiotic resistance for which initial empirical antibiotic therapy might fail (Bobelyte et al., 2017). Resistant to third generation cephalosporins ranged from $6 \%$ for $E$. coli isolates in Uganda (Mugalu et al., 2006) to 48\% in India (Jyothi et al., 2013). Concerning the extended spectrum betalactamase production in Enterobacteriaceae; one reported extended spectrum beta-lactamase production in $65 \%$ of $E$. coli isolates (Jain et al., 2006). In India a study among pediatrics sepsis patients attending Majeedia Hospital of Hamdard University in New Delhi showed various resistance profile of E. coli including: ampicillin (92\%), amoxicillin $(90.9 \%)$, amoxiclav $(68.4 \%)$, cefuroxime $(54.5 \%)$, cefaclor $(84.2 \%)$, cefotaxime $(52.9 \%)$, cefoperazone $(36.8 \%)$, gentamicin $(19 \%)$, amikacin $(12 \%)$, netilmicin $(13 \%)$, ciprofloxacin $(26.1 \%)$, chloramphenicol (16.7\%) and tetracycline (44.4\%) (Alam et al., 2011). In Pakistan Ullah et al., (2016) reported higher level of antibiotic resistance among E. coli isolates including third line antibiotics; Amikacin 58\%, Ciproflaxacin 67.3\%, Enoxacin 83.3\%, Imipenem 94.7\% and Ofloxacin 77.7\%. In Ghana, Obeng-Nkrumah et al. (2016) reported various antibiotic resistance among $E$. coli isolates including ampicillin (97.7\%), amoxicillin clavulanic acid (53.5\%), gentamicin (53.5\%) and ciprofloxacin (62.1\%).

\section{Salmonella spp}

Six out of seven Salmonella species studied were found carrying at least three plasmids of varying sizes. Antimicrobial gene, SulII, was detected in the six Salmonella isolates while aac (3), TetA and blaTEM genes were detected in five Salmonella isolates. One of the Salmonella did not carry any plasmid but showed phenotypic resistance to Cefixime, Ampicillin, Sulfamethoxazole-Trimethoprim and Tetracycline. There was one Salmonella paratyphi B that was multi-resistant to five different antibiotics, three Salmonella paratyphi A were multi-resistant to six different antibiotics. Further, there were two and one Salmonella typhimurium that were multi-resistant to 
five and six different antibiotics respectively. Generally high frequency of resistance was observed for Sulfamethoxazole-Trimethoprim (100\%), Ampicillin (100\%), Tetracycline (100\%), Cefixime (100\%), Gentamycin (100\%) and Ceftriaxone (57.1\%). Studies confirms that the invasive forms of Salmonella disease include enteric fevers (typhoid and paratyphoid fevers) and NTS bacteraemia and are important causes of morbidity and mortality in Asia and Africa (Smith et al., 2014). In India a study among pediatrics sepsis patients attending Majeedia Hospital of Hamdard University in New Delhi showed various resistance profile in Salmonella typhi to various antimicrobials as follows ampicillin (46.4\%), amoxicillin (27.3\%), amoxiclav (15.4\%), cefuroxime $(10 \%)$, cefotaxime $(25 \%)$, cefoperazone $(10.5 \%)$, netilmicin $(10.5 \%)$, ciprofloxacin $(6.3 \%)$, chloramphenicol $(9.1 \%)$ and tetracycline (37\%). Salmonella typhi did not show resistance to gentamicin and amikacin (Alam et al., 2011). On the other hand, in the same study Salmonella paratyphi A did not show resistance to antimicrobials tested (Alam et al., 2011). In Pakistan Ullah et al., (2016) reported higher level of antibiotic resistance among Salmonella spp including third line antibiotics Amikacin 100\%, Ciproflaxacin 66.7\%, Enoxacin 66.7\%, Imipenem $66.7 \%$ and Ofloxacin $83.3 \%$. In Ghana, Obeng-Nkrumah et al. (2016) reported various antibiotic resistance among Salmonella spp including ampicillin $(63.9 \%)$ and amoxicillin clavulanic acid $(23.9 \%)$. In Zanzibar, the majority of the $S$. Typhi isolates $6 / 7$ (86\%) were multidrug-resistant (i.e. resistant to ampicillin, trimethoprim-sulfamethoxazole and chloramphenicol), but susceptible to cefotaxime (Onken et al., 2015).

\section{Pseudomonas spp}

The antimicrobial gene, blaTEM, was detected in five of the Pseudomonas plasmids while four of the plasmids carried SulII, TetA and aac (3) antimicrobial resistance genes. High frequency of resistance was observed for Tetracycline (100\%), Ampicillin (100\%), Cefixime (80\%), Cetriaxone (80\%), Amoxyclavulanic acid $(80.0 \%)$, Gentamicin $(60.0 \%)$ and Sulfamethoxazole-Trimethoprim $(60 \%)$, All the isolates were multiply resistant to between to two to six antibiotics. These results were similar to those in other studies; In India Alam et al., (2011) among pediatrics sepsis patients attending Majeedia Hospital of Hamdard University in New Delhi showed various resistance profile in Pseudomonas spp including: ampicillin (93.8\%), amoxicillin (93.3\%), amoxiclav (90.9\%), cefuroxime $(75 \%)$, cefotaxime $(46.2 \%)$, cefazidime $(38.5 \%)$, cefoperazone $(42.9 \%)$, gentamicin $(33.3 \%)$, amikacin (11.8\%), netilmicin (23.1\%), ciprofloxacin (20\%), ofloxacin $(20 \%)$, chloramphenicol $(40 \%)$ and tetracycline (36.4\%). In Ghana, Obeng-Nkrumah et al. (2016) reported various antibiotic resistance among Pseudomonas spp gentamicin (32.8\%), ampicillin (13.1\%), ciprofloxacin (19.6\%). In Pakistan Mir et al., (2011) reported no resistance to antibiotics including gentamicin among Gram-negative bacteria including Pseudomonas and E. coli. In Vietnam $56 \%$ of the sepsis causing Pseudomonas isolates were resistant to carbapenem resistant (Le et al., 2016). In Turkey, Pseudomonas spp which was the major causative pathogens of sepsis in children was $40.5 \%$ resistant to imipenem (Teke et al., 2016). Evaluation of the trends in antimicrobial resistance of bloodstream infections at a general hospital in Mid-Norway, showed, Pseudomonas spp were $100 \%$ resistant to cefotaxim and $6.9 \%$ to Imipenem (Mehl et al., 2017).

In conclusion, in this geographically defined region of Kenya, of the $33.9 \%$ children with septicemia, gram positive bacteria were the leading cause septicemia. Specifically, S. epidermidis and S. aureus. However, several other Gram-negative bacteria were implicated such as E. coli, P. aeruginosa, $S$. typhimurium and $S$. hemolyticus. It should be noted that gram-negative bacteria have often been implicated in the pathogenesis of severe sepsis and septic shock than the Gram-positive counter parts (Alexandraki and Palacio, 2010). Compared to Gram positive, majority of Gram-negative bacteria were resistant to penicillin (Ampicillins) 100\% tetracycline 96.1\%, sulphonamides (Trimethoprim/sulfamethoxazole) 84.6\%, Aminoglycosides (Gentamicin) 73.1\% and least resistant to Quinolone (Ciprofloxacin) 19.2\%. The Gram-positive bacteria were resistant to sulphonamides (Trimethoprim/sulfamethoxazole) 96.7\%, tetracycline 76.7\%, penicillin (Oxacilline) 73.3\% and Quinolone (Ciprofloxacin) 30\%. The following antimicrobial resistant genes mecA, SulII, blaTEM, TetA aac (3) were identified However, we did not find apparent relationship between carriage of plasmids and antimicrobial resistance.

\section{Acknowledgments:}

We are grateful to the research participants who contributed greatly to this study. Further, we acknowledge laboratory personnel for significant support. Special thanks go to the supervisors for their support and input during various stages of this research project.

\section{Competing interests}

The authors declare no competing interests. 


\section{Authors' contributions}

This work was part of Master of Science degree for GHS in infectious diseases of Kenyatta University. GHS, GG, YI and MK conceived the study. CK participated in the laboratory assays while GHS analyzed the data and prepared the draft manuscript. GHS, GG, YI and MK provided guidance and mentorship during the implementation of the study. All authors reviewed and approved the final manuscript.

\section{Reference}

1. Alam MS, Pillai PK, Kapur P, Pillai KK. Resistant patterns of bacteria isolated from bloodstream infections at a university hospital in Delhi. Journal of Pharmacy \& Bioallied Sciences. 2011;3(4):525530 .

2. Alexandraki I, Palacio C. Gram-negative versus Gram-positive bacteremia: what is more alarmin(g)? Critical Care. 2010;14(3):161.

3. Bataar OA, Lundeg GA, Tsenddorj GA, Jochberger SB, Grander WC, Baelani D, Wilson IE, Baker TF, Dünser WG \& For The Helfen Berührt Study Team. Nationwide survey on resource availability for implementing current sepsis guidelines in Mongolia. Bulletin of The World Health Organization. 2010; 88:839-846

4. Becker K, Heilmann C, Peters G. Coagulase-negative staphylococci. Clin Microbiol Rev. 2014; 27 (4):870-926.

5. Bobelytė O, Gailiūtė I, Zubka V, Žilinskaitė V. Sepsis epidemiology and outcome in the paediatric intensive care unit of Vilnius University Children's Hospital. Acta Medica Lituanica. 2017;24(2):113120.

6. Buehler, S. S. et al. 2016. Effectiveness of Practices To Increase Timeliness of Providing Targeted Therapy for Inpatients with Bloodstream Infections: a Laboratory Medicine Best Practices Systematic Review and Meta-analysis. Clin Microbiol Rev 29, 59-103

7. Cassini, A. et al. 2019. Attributable deaths and disability-adjusted life-years caused by infections with antibiotic-resistant bacteria in the EU and the European Economic Area in 2015: a population-level modelling analysis. Lancet Infect Dis 19, 56-66,

8. David MZ, Daum RS. Community-associated methicillin-resistant Staphylococcus aureus: epidemiology and clinical consequences of an emerging epidemic. Clinical microbiology reviews. 2010; 23(3): 616-87.

9. Dramowski A, Cotton MF, Rabie H, Whitelaw A. Trends in paediatric bloodstream infections at a South African referral hospital. BMC Pediatrics. 2015; 15:33.

10. Ferrer R, Martin-Loeches I, Phillips G, Osborn TM, Townsend S, Dellinger RP, Artigas A, Schorr C, Levy MM. Empiric antibiotic treatment reduces mortality in severe sepsis and septic shock from the first hour: results from a guideline-based performance improvement program. Crit Care Med. 2014;42(8):1749-55.

11. Fleischmann C, Scherag A, Adhikari N, Hartog C, Tsaganos T, Schlattmann P, Angus D, Reinhart K. 2015. Assessment of Global Incidence and Mortality of Hospital-treated Sepsis. Current Estimates and Limitations. Am J Respir Crit Care Med 193, 259-272,

12. Fortuin-de Smidt MC, Singh-Moodley A, Badat R, Quan V, Kularatne R, Nana T, et al. Staphylococcus aureus bacteraemia in Gauteng academic hospitals, South Africa. International journal of infectious diseases: IJID: official publication of the International Society for Infectious Diseases. 2015; 30:41-8.

13. Fuchs, A., Bielicki, J., Mathur, S., Sharland, M. \& Van Den Anker, J. N. 2018. Reviewing the WHO guidelines for antibiotic use for sepsis in neonates and children. Paediatr. Int Child Health38, S3-S15,

14. Goto, M. \& Al-Hasan, M. N. 2013. Overall burden of bloodstream infection and nosocomial bloodstream infection in North America and Europe. Clin Microbiol Infect 19, 501-509,

15. Hochhut B, Lotfi Y, Mazel D, Faruque SM, Woodgate R, Waldor MK. Molecular analysis of antibiotic resistance gene clusters in vibrio cholerae O139 and O1 SXT constins. Antimicrob Agents Chemother. 2001. 45(11):2991-3000.

16. Jain A, Agarwal J, Bansal S. Prevalence of methicillin-resistant, coagulase negative staphylococci in neonatal intensive care units: findings from a tertiary care hospital in India. J Med Microbiol. 2004;53(Pt 9):941-4.

17. Jaktaji R, Mohiti E. Study of Mutations in the DNA gyrase gyrA Gene of Escherichia coli. Iran J Pharm Res. 2010 Winter;9(1):43-8.

18. Jyothi P, Basavaraj MC, Basavaraj PV. Bacteriological profile of neonatal septicemia and antibiotic susceptibility pattern of the isolates. Journal of Natural Science, Biology, and Medicine. 2013;4(2):306309. 
19. Kaasch AJ, Barlow G, Edgeworth JD, Fowler VG Jr., Hellmich M, Hopkins S, et al. Staphylococcus aureus bloodstream infection: a pooled analysis of five prospective, observational studies. The Journal of infection. 2014; 68(3):242-51.

20. Karunakaran R, Tay S, Rahim F, Lim B, Sam I, Kahar-Bador M, Hassan H, Puthucheary S. Ceftriaxone Resistance and Genes Encoding Extended-Spectrum $\beta$-Lactamase among Non-Typhoidal Salmonella Species from a Tertiary Care Hospital in Kuala Lumpur, Malaysia. Jpn. J. Infect. Dis., 65, 433-435, 2012

21. Kelesidis T, Kelesidis I, Rafailidis PI, Falagas ME. Counterfeit or substandard antimicrobial drugs: a review of the scientific evidence. J Antimicrob Chemother. 2007;60(2):214-36.

22. Kissoon $\mathrm{N}$ et al. World Federation of Pediatric Intensive Care and Critical Care Societies: global sepsis initiative. Pediatr Crit Care Med. 2011; 12(5): 494-503.

23. Kumarasamy KK, Toleman MA, Walsh TR, Bagaria J, Butt F, Balakrishnan R, et al. Emergence of a new antibiotic resistance mechanism in India, Pakistan, and the UK: a molecular, biological, and epidemiological study. Lancet Infect Dis. 2010;10(9):597-602.

24. Le NM, Wertheim HF, Phu Dinh Vu, Khanh Khu DN, Le HT, Hoang NG, Vo HT, Lam YM, Vu CT, Nguyen HT, Thai QT, Nilsson LE, Rydell U, Nguyen HV, Nadjm B, Clarkson H, Hanberger H, Larsson M. High prevalence of hospital-acquired infections caused by gram-negative carbapenem resistant strains in Vietnamese pediatric ICUs: A multi-centre point prevalence survey. Chiu. C-H, ed. Medicine. 2016;95(27): e4099.

25. Lee AC, Chandran A, Herbert HK, et al. Treatment of Infections in Young Infants in Low- and MiddleIncome Countries: A Systematic Review and Meta-analysis of Frontline Health Worker Diagnosis and Antibiotic Access. Byass P, ed. PLoS Medicine. 2014;11(10):e1001741.

26. Lemeshow, S., Hosmer, D. K., Klar, J. \& Lwanga, S. K. 1990. World Health Organization. Adequacy of samples size in health studies. www.tbrieder.org/publications/books_english/ lemeshow_samplesize.pdf (accessed February 2019).

27. Macharashvili N, Kourbatova E, Butsashvili M, Tsertsvadze T, McNutt LA, Leonard MK. Etiology of neonatal blood stream infections in Tbilisi, Republic of Georgia. Int J Infect Dis. 2009;13(4):499-505.

28. Macrina F. L., kopecko D.J, Jones K.R, Ayers D.J, McCowen S.M. 1978. A multiple plasmidcontaining Escherichia coli strain: convenient source of size reference plasmid molecules. Plasmid 1:417-420.CrossRefMedline

29. Marchant EA, Boyce GK, Sadarangani M, Lavoie PM. Neonatal sepsis due to coagulase-negative staphylococci. Clin Dev Immunol. 2013; 2013:586076.

30. Mempel M, Feucht H, Ziebuhr W, Endres M, Laufs R, Gruter L. Lack of mecA transcription in slimenegative phase variants of methicillin- resistant Staphylococcus epidermidis. Antimicrob Agents Chemother 1994; 38:1251-5.

31. Mir F, Tikmani S, Shakoor S, Warraich H, Sultana S, Ali S, Zaidi A (2011) Incidence and etiology of omphalitis in Pakistan: a community-based cohort study. The Journal of Infection In Developing Countries 5 (12): 828-833.

32. Mugalu J, Nakakeeto MK, Kiguli S, Kaddu-Mulindwa DH. Aetiology, risk factors and immediate outcome of bacteriologically confirmed neonatal septicaemia in Mulago hospital, Uganda. Afr Health Sci. 2006;6(2):120-6.

33. Nathan C, Cars O. Antibiotic resistance-problems, progress, and prospects. N Engl J Med. 2014;371(19):1761-3.

34. Obeng-Nkrumah N, Twum-Danso K, Krogfelt K, Newman M. High Levels of Extended-Spectrum Beta-Lactamases in a Major Teaching Hospital in Ghana: The Need for Regular Monitoring and Evaluation of Antibiotic Resistance. Am. J. Trop. Med. Hyg., 89(5), 2013. 960-964

35. Onken A, Said AK, Jørstad M, Jenum PA, Blomberg B (2015) Prevalence and Antimicrobial Resistance of Microbes Causing Bloodstream Infections in Unguja, Zanzibar. PLoS ONE 10(12): e0145632

36. Perez-Perez FJ, Hanson ND. Detection of plasmid-mediated AmpC beta-lactamase genes in clinical isolates by using multiplex PCR. J Clin Microbiol. 2002; 40:2153-62

37. Raad I, Alrahwan A, Rolston K. Staphylococcus epidermidis: Emerging Resistance and Need for Alternative Agents. Clinical Infectious Diseases 1998; 26:1182-7

38. Reinhart, K. Daniels R, Kissoon N, Machado R, Schachter R, Finfer S. 2017. Recognizing Sepsis as a Global Health Priority - A WHO Resolution. New England Journal of Medicine 377, 414-417

39. Şahin M, Unver A, Otlu S. Isolation and Biotyping of Brucella Melitensis from aborted Sheep Fetuses In Turkey. Bull Vet Inst Pulawy 52, 59-62, 2008

40. Sambrook, J. \& Russell, D. (2001). Molecular Cloning: a Laboratory Manual, 3rd edn. Cold Spring Harbor, NY: Cold Spring Harbor Laboratory. 
41. Seale AC, Blencowe H, Zaidi A, et a. Neonatal severe bacterial infection impairment estimates in South Asia, sub-Saharan Africa, and Latin America for 2010. Pediatr Res 2013; 74:73-85

42. Sepsis Alliance. Sepsis fact sheet. https://www.sepsis.org/downloads/2016 sepsis facts media.pdf. Accessed May 5, 2020.

43. Shitaye D, Asrat D, Woldeamanuel Y, Worku B. Risk factors and etiology of neonatal sepsis in Tikur Anbessa University Hospital, Ethiopia. Ethiop Med J. 2010;48(1):11-21.

44. Smith AM, Mthanti MA, Haumann C, Tyalisi N, Boon GPG, Sooka A, et al. Nosocomial outbreak of Salmonella enterica serovar Typhimurium primarily affecting a pediatric ward in South Africa in 2012. J Clin Microbiol. 2014 Feb; 52(2):627 \pm 31.

45. Standards for Antimicrobial Disk Susceptibility Tests; Approved Standard - 10th edition. CLSI Document M02 - A10. Clinical Laboratory Standards Institute (CLSI) 940 Wayne, PA, U.S.A. 2018

46. Szczepanowski R, Linke B, Krahn I, Gartemann H, Gu tzkow T, Eichler W, Pu hler A Schlu ter P. Detection of 140 clinically relevant antibiotic- resistance genes in the plasmid metagenome of wastewater treatment plant bacteria showing reduced susceptibility to selected antibiotics. Microbiology (2009), 155, 2306-2319

47. Talbert AW, Mwaniki M, Mwarumba S, Newton CR, Berkley JA. Invasive bacterial infections in neonates and young infants born outside hospital admitted to a rural hospital in Kenya. Pediatr Infect Dis J. 2010;29(10):945-9.

48. Taxt, A.M., Avershina, E., Frye, S.A. et al. Rapid identification of pathogens, antibiotic resistance genes and plasmids in blood cultures by nanopore sequencing. Sci Rep 10, 7622 (2020).

49. Teke TA, Tanır G, Bayhan G, Öz F, Metin O, Özkan S. Clinical and microbiological features of resistant gram-negative blood stream infections in children. Journal of Infection and Public Health (2017) $10,211-218$

50. Ullah O, Khan A, Ambreen A, Ahmad I, Akhtar T, Gandapor AJ, Khan AM. Antibiotic Sensitivity pattern of Bacterial Isolates of Neonatal Septicemia in Peshawar, Pakistan. Arch Iran Med. 2016; 19(12): $866-869$

51. Vassallo M, Dunais B, Roger PM. Antimicrobial lock therapy in central-line associated bloodstream infections: a systematic review. Infection. 2015; 43(4):389-98.

52. Weill M, Malcolm C, Chandre F, Mogensen K, Berthomieu A, Marquine M, Raymond M. The unique mutation in ace-1 giving high insecticide resistance is easily detectable in mosquito vectors. Insect Mol Biol. 2004. 13(1):1-7.

53. WHO. 2014. Antimicrobial resistance: global report on surveillance. 2014. [http://www.who.int/drugresistance/documents/surveillancereport/en/] accessed August 2017

54. Wielders CL, Fluit AC, Brisse S, Verhoef J, Schmitz FJ. mecA gene is widely disseminated in Staphylococcus aureus population. J Clin Microbiol. 2002 Nov;40(11):3970-5.

55. Zaidi A, Thaver D, Ali SA, Khan TA. Pathogens Associated with Sepsis in Newborns and Young Infants in Developing Countries. Pediatr Infect Dis J 2009;28: S10 